\section{Atención a la salud y morbimortalidad materna: un estudio de casos y controles de base hospitalaria en dos regiones de Colombia (Bogotá y Antioquia), 2009-2011}

\section{Healthcare and maternal morbidity and mortality: a hospital-based case-control study in two regions of Colombia (Bogotá and Antioquia), 2009-2011}

Atenção à saúde e morbimortalidade materna: estudo caso-controle de base hospitalar em duas regiões de Colômbia (Bogotá e Antioquia), 2009-2011

\section{Resumen}

El objetivo fue identificar si formas de pago y regímenes de aseguramiento están asociados con la mortalidad materna y morbilidad obstétrica extrema. Estudio de casos y controles de base hospitalaria en dos regiones de Colombia, 2009-2011. Los datos se obtuvieron de la historia clínica de cada gestante. Se utilizó regresión logística no condicional. El resultado fue: 1.011 pacientes, 337 casos y 674 controles. Ningún componente de calidad fue estadísticamente significativo en ambas regiones. En Bogotá, el riesgo de complicación obstétrica, significativamente mayor en Régimen Contributivo que en Subsidiado y no aseguradas; Antioquia, aunque hubo asociaciones similares, no estadísticamente significativas. Diferencias en morbilidad por régimen de pago no estadísticamente significativas en Antioquia ni Bogotá. Factores asociados a la morbimortalidad materna diferentes, según la población estudiada, lo que sugiere la necesidad de estudios locales para identificar factores determinantes propios y tomar decisiones pertinentes.

Seguro de Salud; Complicaciones del Embarazo; Mortalidad Materna
Francisco J. Yepes 1 Joaquin G. Gómez 2 John Jairo Zuleta 2 Juan Luis Londoño 1 Jorge Luis Acosta-Reyes 3 Luz Helena Sánchez-Gómez 1 Marta L. Ramírez 1

doi: 10.1590/0102-311X00080215
1 Asociación Colombiana de la Salud, Bogotá, Colombia. 2 Centro Nacer, Salud Sexual y Reproductiva, Medellín, Colombia.

3 Departamento de Salud Pública, Universidad del Norte, Barranquilla, Colombia.

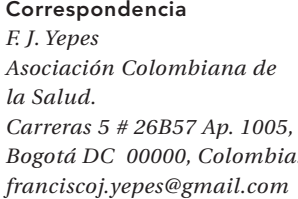




\section{Introducción}

La mortalidad materna y la morbilidad obstétrica extrema son problemas de salud pública en los países en vía de desarrollo, en donde ocurren el 99\% de las muertes maternas que se presentan en el mundo 1. La morbilidad obstétrica extrema es una complicación que requiere una intervención médica urgente encaminada a prevenir la muerte de una materna ${ }^{2}$, por su parte, la mortalidad es la punta del iceberg del fenómeno, pues cada deceso, representa una mayor cantidad de mujeres que sufren complicaciones; muchas de ellas, con secuelas a corto y largo plazo 2,3,4,5. La morbilidad obstétrica extrema es un indicador complementario a la razón de mortalidad materna (RMM), pues permite un análisis más amplio del problema, involucrando un mayor número de pacientes para obtener conclusiones más robustas, identifica aspectos positivos que evitaron un desenlace fatal, permite evaluar el desempeño de los sistemas y servicios de salud y obtiene información directa de la mujer que tuvo la complicación y estuvo en riesgo de fallecer 4,6,7,8

El exceso de mortalidad materna en los países en desarrollo, adicional a algunos factores de riesgo biológicos, se explica por la presencia de factores de riesgo no biológicos, como la pobreza, la baja educación, la falta de cobertura del parto institucional, la falta de insumos y recursos, la falta de tecnología y factores propios de cada país. Aunque Colombia es un país con altas coberturas de parto institucional (95,3\%), de controles prenatales (96\%) ${ }^{9} \mathrm{y}$ con un importante grado de desarrollo de infraestructura hospitalaria y tecnológica, esto no se refleja en las RMM o de morbilidad obstétrica externa 10 , que siguen siendo elevadas.

En Colombia existe un sistema de aseguramiento en salud que distribuye su población en regímenes, de acuerdo a su capacidad de aportar dinero al sistema. $\mathrm{Al}$ régimen contributivo pertenecen las personas que trabajan y aportan económicamente al sistema; al subsidiado, personas sin relación laboral ni capacidad de pago, por quienes el sistema responde, mediante un proceso de identificación y subsidio estatal. Para el año 2011, según cifras oficiales, en Antioquia el 51,9\% de la población pertenecía al régimen contributivo y el $38 \%$ al subsidiado; las respectivas cifras en Bogotá eran 72\%, 17,5\%. Un tercer grupo no tiene afiliación a estos regímenes y se conoce como la población pobre no asegurada, popularmente denominada vinculada, en Antioquia correspondía al $10 \%$ y en Bogotá al 10,5\%. Las personas no afiliadas no han reunido los requisitos de pobreza requeridos y tampoco tienen recursos para incorporarse el régimen contributivo. Deben acceder a la red pública directamente. Cada uno de estos regímenes poseía a la fecha del estudio un plan de beneficios diferente, siendo el más completo el del régimen contributivo. Esta distribución limita los servicios a los que pueden acceder las personas, lo que crea diferencias en la atención en salud.

Bogotá y Antioquia son los dos conglomerados humanos más densos del país. Según cifras de vigilancia epidemiológica del año 2008, antes de iniciar esta investigación, en Antioquia, la población del régimen subsidiado presentó una razón de mortalidad de 62 por 100 mil nacidos vivos, superior a la del contributivo con una razón de 25, en tanto que en Bogotá la población del régimen subsidiado tuvo una razón de 43 por 100 mil nacidos vivos, mayor que la de los otros regímenes.

Por otro lado, en Colombia existen diferencias en los mecanismos de pago por la prestación de los servicios de salud entre las aseguradoras y las instituciones prestadoras de servicios de salud, que están definidas por el Decreto no 4.747 de 2007 11. El pago por capitación está basado en el concepto de enfermo potencial, y es un pago anticipado de una suma fija de dinero para la atención de todos los eventos que un individuo pueda presentar en un periodo de tiempo. Este mecanismo de pago implica que la instituciones prestadoras de servicios de salud asumen el riesgo económico y el compromiso de hacer actividades de promoción de la salud, para prevenir enfermedades que se consuman el pago por capitación. Sin embargo, este mecanismo de pago puede conducir a "estímulos perversos” como la selección de los usuarios más sanos (descreme o cream skimming) y el racionamiento en el uso de los servicios 12 . El pago por evento es otro mecanismo, en el cual el pago se realiza por las actividades realmente prestadas a un paciente durante un período determinado de tiempo e implica la responsabilidad económica del asegurador. Igualmente, esta forma de pago genera otro tipo de "incentivos perversos" como la inducción de oferta innecesaria de servicios. Según el interés del prestador o del asegurador, reflejado en el mecanismo de pago, es posible que los servicios se presten de forma diferencial, lo cual puede llevar a deficiencias en la atención de las mujeres en embarazo, y ser un factor de riesgo para el desarrollo de una mortalidad materna o una morbilidad obstétrica externa. El objetivo general de esta investigación fue identificar si los mecanismos de pago y los regímenes de aseguramiento se asocian con un aumento de la probabilidad de sufrir una mortalidad materna o una morbilidad obstétrica externa en mujeres hospitalizadas de Bogotá y Antioquia, Colombia. 


\section{Métodos}

Estudio de casos y controles equiparado por tipo de enfermedad causante de la morbilidad obstétrica externa (pre eclampsia, hemorragia y sepsis). Se implementó un modelo de casos y controles, porque las complicaciones graves en las gestantes son poco frecuentes y se hizo un equiparamiento para evitar comparar condiciones clínicas disímiles entre sí; de esta forma, se genera una categoría nueva que es "complicación obstétrica” y quedan subyacentes las condiciones clínicas particulares, en estas condiciones cada caso queda ligado a un control de la misma enfermedad, con lo que se controlan las potenciales diferencias entre las enfermedades evaluadas. La cohorte hipotética para la selección de los casos y controles estuvo conformada por las mujeres embarazadas con preeclampsia, hemorragia o sepsis, atendidas en los hospitales de segundo y tercer nivel de complejidad del departamento de Antioquia y en la ciudad de Bogotá DC, Colombia, que en los años previos al inicio del estudio hubieran atendido más de 1.000 partos por año. Para Antioquia se incluyeron 7 instituciones de segundo nivel y 11 de tercer nivel de atención, que atienden el 80,6\% de los partos del Departamento. Estas instituciones atienden tanto personas afiliadas a los diferentes regímenes de afiliación al sistema de salud, como no afiliadas, en cuyo caso facturan los costos de la atención a los entes territoriales de salud. En Bogotá sólo dos hospitales de segundo o tercer nivel no se incluyeron por tener menos de 1.000 partos al año. Los hospitales incluidos atendieron el $82 \%$ de los partos.

Entraron en el estudio todas las mujeres con embarazo único o múltiple o en posparto y más de 22 semanas de gestación, atendidas entre el primero de enero de 2009 y el 31 de diciembre de 2011 en hospitales de Bogotá y Antioquia. Se definió como caso la paciente que presentó preeclampsia, hemorragia o infección con alguno de los criterios de morbilidad obstétrica externa, definidos en el protocolo del estudio (Tabla 1); por su parte, se eligieron como control mujeres con la misma enfermedad que los casos, pero sin los criterios de morbilidad obstétrica externa, y que fueran atendidas en el mismo hospital y en el mismo periodo que los casos.

La identificación de las mujeres se hizo de manera prospectiva y estuvo a cargo del personal de enfermería de cada hospital que fue entrenado al inicio de la investigación. Estas enfermeras generaron un listado de casos consecutivos con todas las mujeres a quienes los médicos tratantes, no integrantes del equipo de investigación, asignaban uno de los tres diagnósticos en estu- dio. Dos veces por semana los médicos especialistas en obstetricia encargados del proceso de vigilancia epidemiológica oficial, no integrantes del equipo de investigación, revisaban las historias clínicas de las mujeres elegibles y diligenciaban un instrumento estructurado con todos los datos preestablecidos para la investigación. La verificación de los criterios para el caso y sus controles fue hecha por el grupo de investigación en el momento de analizar cada historia clínica.

La información se tomó según los diagnósticos registrados en la historia clínica por los médicos tratantes. Las variables regímenes de aseguramiento y mecanismo de pago fueron solicitadas directamente a cada una de las instituiciones prestadoras de servicios de salud, y definidas según los criterios del Decreto no 4.747 de 200711. La calidad de la atención fue definida por el Comité de Vigilancia Epidemiológica (COVE) de cada región (Bogotá y Antioquia), conformado por obstetras con experiencia en el análisis de muerte materna. Para la calificación de la calidad de la atención se tuvieron en cuenta el diagnóstico y el tratamiento oportunos y correctos. Para calificar la oportunidad, el COVE tuvo en cuenta el tiempo empleado para, a partir de los criterios consignados en la historia, obtener un diagnóstico y, después de contar con él, instaurar un tratamiento. Lo correcto se refiere a la coherencia, verificable en la historia clínica, del diagnóstico con los hallazgos clínicos y paraclínicos y, para el tratamiento, el cumplimiento con los protocolos de atención vigentes basados en la mejor evidencia. Con esto se obtuvieron cuatro criterios independientes de calidad: diagnóstico oportuno y correcto, y tratamiento oportuno y correcto. Éstos se utilizaron de manera independiente en los análisis o se agregaron en una sola variable denominada calidad de la atención, esta se consideraba buena si los cuatro ítems habían sido calificados simultáneamente como correctos (sin fallas), y se consideraba falta de calidad, si se fallaba al menos en uno de ellos (al menos una falla). Aunque estos juicios tienen un grado de subjetividad, las decisiones se tomaron en conjunto en el COVE. Este consenso implica una revisión y discusión amplia de la historia clínica, confrontación contra el conocimiento de los expertos participantes y contra las guías de práctica clínica, búsqueda y actualización de la literatura, cuando la situación lo amerita y argumentación entre los asistentes. No se realiza proceso formal de votación sino que, después de este proceso, se toman decisiones en común acuerdo. Para verificar la concordancia en los análisis entre los dos comités se utilizó el estadístico de kappa, se seleccionó al azar un 10\% de los registros. Se hizo evaluación cruzada, es decir, cada COVE evaluó 
Tabla 1

Definición de morbilidad obstétrica extrema según evento mórbido de inclusión, Bogotá DC y Antioquia, Colombia, 2011/2012.

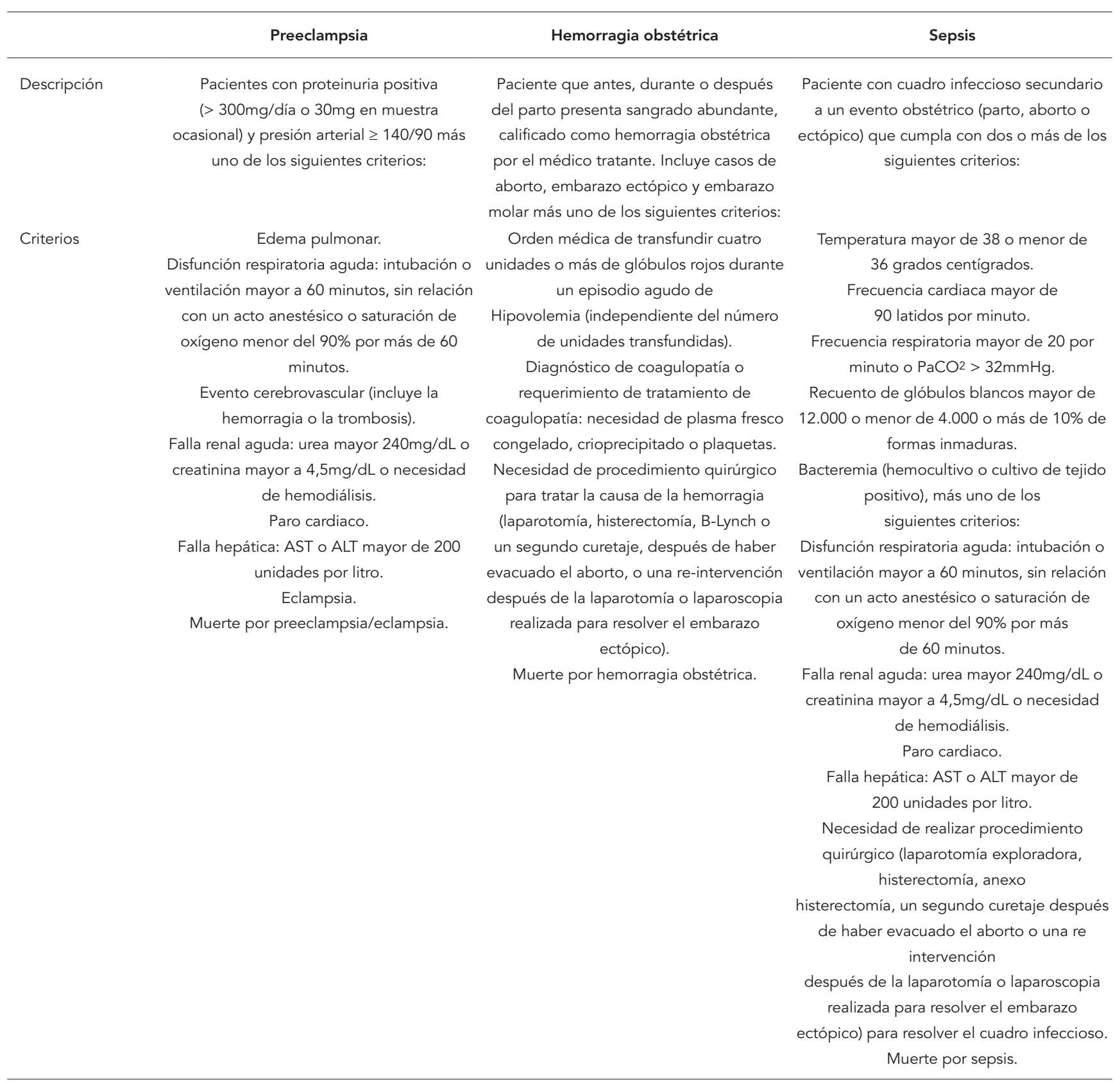

los registros que previamente había analizado el otro, sin conocer los juicios previos y el resultado fue de 0,8 , indicando muy buena concordancia interobservador.

Se incluyeron para el análisis variables sociodemográficas, antecedentes obstétricos y patológicos, número de controles prenatales, problemas administrativos relacionados con dificultades para el transporte y falta de insumos. Los datos se obtuvieron de la historia clínica com- pleta (electrónica o manual) de cada gestante en cada institución. Dentro de los antecedentes patológicos se incluyeron las condiciones clínicas que al momento de elaborar el protocolo se consideró que pudieran afectar la evolución de las pacientes y, para identificarlas, se respetó el criterio clínico del médico tratante consignado en las historias clínicas. Se tuvieron en cuenta la diabetes, que incluye diabetes preconcepcional y diabetes gestacional, hipertensión arterial, en- 
fermedad cardiovascular, enfermedades del colágeno (todas las posibles), la infección urinaria durante el embarazo actual, enfermedad mental y una categoría de otras, en las que se podían incluir neoplasias, enfermedades renales, hepáticas, pulmonares o endocrinológicas presentes durante la gestación en estudio, independiente de su momento de aparición con respecto al embarazo, del grado de control o de su condición de estar o no recibiendo tratamiento. Para los análisis, estas condiciones clínicas se agregaron para generar una variable dicotómica, ausencia de cualquiera de ellas ("sin enfermedades crónicas") o presencia de al menos una de ellas ("al menos una”). El índice de masa corporal tenido en cuenta fue el consignado en la historia clínica antes de las 16 semanas de gestación.

Las variables cuantitativas se categorizaron de acuerdo al riesgo que implica tener uno u otro número. El número de semanas al primer control se categorizó en tres grupos, antes de las 13 semanas, entre 12 y 20 semanas, y después de 20 semanas. Las mujeres sin consulta de control prenatal -o con menos de cuatro- tienen menor posibilidad de que se les detecte e intervengan sus posibles riesgos, comparado con las de más de cuatro, por lo tanto el número de controles se dividieron en esos tres grupos. El período integenésico de 2 o menos años incrementa los riesgos del embarazo, por lo tanto este tiempo se dividió según este límite. De manera similar, se categorizaron la edad, el número de gestaciones, de abortos y el índice de masa corporal.

Se calculó un tamaño muestral que permitiera detectar un odds ratio (OR) de 2,0 para el mecanismo de pago de la atención en salud como factor de riesgo para morbilidad obstétrica externa, asumiendo probabilidades de errores tipo I y II de 0,05 y 0,20, respectivamente. Éste se aumentó en un $10 \%$ previendo algunas pérdidas de casos y controles. En Bogotá se tuvo en cuenta que la mortalidad materna es mayor en el régimen subsidiado, con una proporción de $23,2 \%$, y en Antioquia la mortalidad materna era mayor para la población no asegurada con un $12,7 \%$. Por cada caso se seleccionaron, de manera aleatoria, 2 controles a partir del listado de potenciales controles, de forma que se obtuvieron tripletas de mujeres para cada una de las tres enfermedades estudiadas en la investigación. Con estas proporciones se definieron tamaños muestrales de 141 y 200 casos para Bogotá y Antioquia respectivamente, para un total de 341 casos y 682 controles ${ }^{13}$. Debido a este conocimiento previo, desde el protocolo se planeó evaluar si efectivamente estas dos regiones tenían comportamientos diferentes y, según eso, se tomaría la decisión de presentar un análisis general o uno discriminado por región.
Se presentan las frecuencias relativas y absolutas para las variables cualitativas. Se evaluó el supuesto de normalidad para las variables cuantitativas y, según ello, se presentan como medias y desviación estándar o medianas y cuartiles. Para establecer asociaciones entre las variables categóricas con la variable dependiente se emplearon las pruebas de distribución aproximada chi ${ }^{2}$ o exacta de Fisher, según la frecuencia observada, con niveles de significación del 5\%. La hipótesis de trabajo fue unidireccional, y se obtuvieron estimaciones de las asociaciones entre los diferentes factores de riesgo o protectores y la morbilidad obstétrica externa, que se expresan en OR con sus respectivos intervalos de confianza del 95\% (IC95\%).

Con el fin de obtener las estimaciones ajustadas, se utilizó inicialmente un modelo de regresión logística condicional; los resultados obtenidos no diferían de los obtenidos mediante la regresión logística no condicional y, por consiguiente, se decidió utilizar esta última. En el modelo de regresión utilizado se incluyeron todas las variables que tuvieran significación estadística con excepción de zona rural- urbana en Bogotá y diabetes, debido a su bajo número, y las que tuvieran importancia clínica, con el fin de obtener OR's ajustadas, modelo utilizado cuando el propósito es controlar efectos de confusión (método "enter") Todos los cálculos se realizaron con el paquete estadístico SPSS, versión 17 (SPSS Inc., Chicago, EE.UU.).

Los Comités de Bioética de la Facultad de Medicina de la Universidad de Antioquia y de Assalud aprobaron el protocolo de investigación previo a la ejecución. Se contó además con la aprobación de cada comité de investigación de los hospitales participantes de Antioquia. En Bogotá el estudio estuvo anidado en el proceso de vigilancia epidemiológica de la Secretaria Distrital de Salud.

\section{Resultados}

Entre enero de 2009 y diciembre de 2011 se seleccionaron 1.011 pacientes: 337 casos y 674 controles. Según el tipo de morbilidad se identificaron 477 (47,2\%) mujeres con pre eclampsia (159 casos y 318 controles), 381 (37,7\%) con hemorragia obstétrica (127 casos y 254 controles) y 153 (15,1\%) con sepsis obstétrica 51 casos y 102 controles). Hubo cuatro muertes, 3 por hemorragia y una por preeclampsia. Debido a grandes diferencias en las características de las pacientes y las condiciones de la atención entre Antioquia y Bogotá, se presentan los resultados de manera independiente, según lo planeado en el protoco- 
lo. En el análisis estadístico se confirmó que el sitio de residencia y sus características ejercían un efecto de interacción sobre los resultados, por lo tanto la forma correcta de presentarlos es discriminada para cada región. La Tabla 2 presenta las características de las pacientes incluidas en el estudio, diferenciadas según su condición de casos o controles.

La forma de pago más frecuentemente utilizada fue el pago por evento (Tabla 2); en Antioquia el $94 \%$ de los casos y $98,2 \%$ de los controles, y en Bogotá, el $69,3 \%$ de los casos y el $67 \%$ de los controles.

La Tabla 3 presenta las medidas de asociación ajustadas que se obtuvieron para las características clínicas, demográficas y de la atención con la morbilidad obstétrica externa en Antioquia y Bogotá. No se encontró asociación estadística entre la forma de pago y el riesgo de sufrir una mortalidad obstétrica externa ni en Bogotá OR = 1,3 (IC95\%: 0,8-2,1), ni en Antioquia OR = 0,05 (IC95\%: 0,0-1,1).

Como se observa en los resultados que figuran en la Tabla 3, ninguno de los cuatro componentes de la calidad de la atención médica resultó ser estadísticamente significativo en ambas regiones. En Bogotá, de acuerdo con la aproximación que ofrece la OR, el riesgo de una complicación obstétrica, resultó ser significativamente mayor en el Régimen Contributivo que en el Régimen Subsidiado y en las pacientes no aseguradas; en Antioquia, en cambio, aunque se observaron asociaciones en el mismo sentido, ellas no fueron estadísticamente significativas. Las diferencias en la morbilidad obstétrica por régimen de pago no fueron estadísticamente significativas ni en Antioquia ni en Bogotá.

\section{Discusión}

Las hipótesis de trabajo fueron que el mecanismo de pago por capitación y la afiliación al régimen subsidiado estaban asociados con la aparición de una morbilidad obstétrica externa. No se encontró asociación con el pago por capitación en ninguna de las dos regiones, y se observó que el régimen subsidiado y el no aseguramiento se comportaron como factores protectores para la morbilidad obstétrica externa en Bogotá y no tuvieron efecto en Antioquia. En general, la experiencia en otros países es la del aseguramiento, actuando como factor protector, pero no conocemos de experiencias similares a los regímenes colombianos de aseguramiento 14,15. Estos hallazgos, en contraste con los hallazgos de García 16 en el control prenatal, se pueden explicar porque el personal de la salud no discrimina por régimen de afiliación cuando brinda su proceso de atención, en otras palabras la atención no está determinada por el régimen de afiliación por consiguiente es congruente con los hallazgos en las dos regiones, en cuanto al sistema de contratación. Por otro lado la Secretaria Distrital de Salud de Bogotá brinda una asesoría y asistencia técnica muy rigurosa a las instituiciones prestadoras de servicios de salud que atienden las poblaciones subsidiada y no asegurada (instituciones públicas), situación que no ocurre con las instituciones que atienden la población del régimen contributivo (instituciones privadas), las cuales se autorregulan, esto podría ayudar a explicar el porqué de la diferencia encontrada en la morbilidad obstétrica externa entre regímenes de afiliación en Bogotá. En Antioquia a diferencia de la capital, las pacientes del régimen subsidiado o las no aseguradas son atendidas en las mismas instituciones donde se atienden las pacientes del régimen contributivo y, por otro lado, la Secretaría de Salud del departamento brinda asesoría y asistencia técnica, vigilancia y control a todas las instituciones, independientemente de su naturaleza pública o privada. Estos hallazgos sugieren la necesidad de no segmentar los procesos de las secretarías de salud, ni tampoco las redes de prestación de servicios y ejercer las funciones en igualdad de condiciones para todo el sistema de salud, independiente de régimen de afiliación y de la naturaleza de las instituciones.

Estos hallazgos refuerzan la necesidad de implementar a cabalidad la Ley no 1.438 de 201117 y la ley estatutaria de salud de 2015, donde se plasma claramente la salud como un derecho de la población y la necesidad de un sistema de salud basado en la atención primária de la salud y la necesidad de conformar redes integradas de servicios de salud 17,18. Se documentó deficiencia en la calidad de la atención en toda la población de mujeres incluidas en la investigación. Hubo al menos una falla en la atención en una tercera parte de las mujeres en Antioquia y en la mitad de las de Bogotá. Los problemas de falta de calidad existen tanto en países desarrollados como en vías de desarrollo. En el estudio de hemorragia obstétrica grave de Bouvier-Colle et al. 19 hubo problemas de calidad de la atención en un $38 \%$ de las mujeres con morbilidad obstétrica externa, y en el análisis de muertes maternas en Inglaterra en el período 2003-2005 fueron del $64 \% 20$ y para el período $2006-2008$ del $70 \% 21$.

$\mathrm{Si}$ bien lo anterior es una realidad, es importante resaltar que en Antioquia la RMM es muy superior a la meta planteada por el Instituto $\mathrm{Na}$ cional de Salud 22 y aún mayor que la razón de los países desarrollados, lo que implica que debemos hacer mayores esfuerzos en el cuidado 
Tabla 2

Características demográficas y clínicas de la población en estudio.

\begin{tabular}{|c|c|c|c|c|c|c|}
\hline \multirow[t]{2}{*}{ Características } & \multicolumn{2}{|c|}{ Antioquia (\%) [n = 588] } & \multirow{2}{*}{$\begin{array}{l}\text { Valor de p } \\
\text { unilateral }\end{array}$} & \multicolumn{2}{|c|}{ Bogotá (\%) [n = 423] } & \multirow{2}{*}{$\begin{array}{l}\text { Valor de p } \\
\text { unilateral }\end{array}$} \\
\hline & $\begin{array}{c}\text { Casos } \\
{[n=196]}\end{array}$ & $\begin{array}{l}\text { Controles } \\
{[n=392]}\end{array}$ & & $\begin{array}{l}\text { Casos } \\
{[n=141]}\end{array}$ & $\begin{array}{l}\text { Controles } \\
{[n=282]}\end{array}$ & \\
\hline Zona de residencia urbana & $163(83,2)$ & $361(92,1)$ & 0,01 & $139(98,6)$ & $281(99,6)$ & 0,18 \\
\hline Edad años [media (DE)] & $27,03(7,7)$ & $26,10(7,1)$ & 0,08 & $27(6,6)$ & $25,9(7,2)$ & 0,08 \\
\hline Estado civil & & & 0,23 & & & 0,34 \\
\hline Casada & $59(30,3)$ & $109(27,9)$ & & $28(19,9)$ & $47(16,7)$ & \\
\hline Soltera & $62(31,8)$ & $133(34,0)$ & & $38(27,0)$ & $71(25,2)$ & \\
\hline Unión libre + otro & $74(37,9)$ & $149(38,1)$ & & $75(53,2)$ & $164(58,2)$ & \\
\hline Régimen de aseguramiento & & & 0,98 & & & 0,00 \\
\hline Contributivo + especial & $103(53,1)$ & $201(51,5)$ & & $75(53,2)$ & $106(37,6)$ & \\
\hline Subsidiado & $66(34,0)$ & $139(35,6)$ & & $37(26,2)$ & $98(34,8)$ & \\
\hline No asegurado & $25(12,9)$ & $50(12,8)$ & & $29(20,6)$ & $78(27,7)$ & \\
\hline Educación de la gestante * & & & 0,01 & & & 0,00 \\
\hline Primaria & $26(24,8)$ & $45(24,7)$ & & $20(14,2)$ & $20(7,1)$ & \\
\hline Secundaria & $34(32,4)$ & $81(44,5)$ & & $75(53,2)$ & $186(66,0)$ & \\
\hline Técnica o universitaria & $45(42,9)$ & $56(30,8)$ & & $46(32,6)$ & $76(27,0)$ & \\
\hline Forma de pago & & & 0,02 & & & 0,32 \\
\hline Capitación & $12(6,1)$ & $7(1,8)$ & & $43(30,7)$ & $93(33,0)$ & \\
\hline Evento & $184(93,9)$ & $385(98,2)$ & & $97(69,3)$ & $189(67,0)$ & \\
\hline Abortos previos & & & 0,22 & & & 0,4 \\
\hline Sin antecedente & $152(77,6)$ & $309(78,8)$ & & $110(78,0)$ & $217(77,0)$ & \\
\hline 1 & $29(14,8)$ & $58(14,8)$ & & $25(17,7)$ & $49(17,4)$ & \\
\hline 2 o más & $15(7,7)$ & $25(6,4)$ & & $6(4,3)$ & $16(5,7)$ & \\
\hline Partos previos & & & 0,19 & & & 0,06 \\
\hline Nulípara & $103(52,6)$ & $210(53,6)$ & & $62(44,0)$ & $153(54,3)$ & \\
\hline Primíparas & $40(20,4)$ & $94(24,0)$ & & $49(34,8)$ & $77(27,3)$ & \\
\hline Al menos un parto & $53(27,0)$ & $88(22,4)$ & & $30(21,3)$ & $52(18,4)$ & \\
\hline Periodo intergenésico ** & & & 0,26 & & & 0,31 \\
\hline Mayor de 2 años & $96(91,4)$ & $192(90,6)$ & & $59(64,8)$ & $101(66,0)$ & \\
\hline 2 años o menos & $9(8,6)$ & $20(9,4)$ & & $32(35,2)$ & $52(34,0)$ & \\
\hline Controles prenatales & & & 0,21 & & & 0,06 \\
\hline Sin control & $33(17,7)$ & $59(15,7)$ & & $42(29,8)$ & $87(30,9)$ & \\
\hline 1-4 controles & $37(19,9)$ & $74(19,7)$ & & $20(14,2)$ & $28(9,9)$ & \\
\hline Más de 4 controles & $116(62,4)$ & $243(64,6)$ & & $79(56,0)$ & $167(59,2)$ & \\
\hline $\begin{array}{l}\text { Numero de semanas al primer control } \\
\text { (semanas) }\end{array}$ & & & 0,32 & & & 0,16 \\
\hline Antes de las 13 & $41(25,3)$ & $122(36,7)$ & & $92(65,2)$ & $190(67,6)$ & \\
\hline Entre 13-20 & $14(8,6)$ & $41(12,3)$ & & $36(25,5)$ & $59(21,0)$ & \\
\hline Después de las 20 & $107(66,0)$ & $169(50,9)$ & & $13(9,2)$ & $32(11,4)$ & \\
\hline Diabetes & $5(2,6)$ & $2(0,5)$ & 0,03 & $0(0,0)$ & $5(1,8)$ & 0,12 \\
\hline Hipertensión arterial & $11(5,6)$ & $30(7,7)$ & 0,30 & $10(7,1)$ & $13(4,6)$ & 0,27 \\
\hline Enfermedad cardiovascular & $1(0,5)$ & $0(0,0)$ & 0,18 & $2(4,3)$ & $2(0,7)$ & 0,38 \\
\hline Enfermedad del colágeno & $1(0,5)$ & $1(0,3)$ & 0,30 & $6(4,3)$ & $2(0,7)$ & 0,01 \\
\hline Infección urinaria & $7(3,6)$ & $9(2,3)$ & 0,18 & $50(35,5)$ & $116(41,1)$ & 0,13 \\
\hline Enfermedad mental & $0(0,0)$ & $2(0,5)$ & 0,60 & $3(2,1)$ & $2(0,7)$ & 0,12 \\
\hline Otras enfermedades & $41(21,0)$ & $75(19,2)$ & 0,30 & $16(11,3)$ & $30(10,6)$ & 0,36 \\
\hline Presencia de enfermedad crónica & & & 0,42 & & & 0,14 \\
\hline Sin enfermedades crónicas & $179(92,3)$ & $360(91,8)$ & & $68(48,2)$ & $140(49,6)$ & \\
\hline Al menos una & $15(7,7)$ & $32(8,2)$ & & $73(51,8)$ & $142(50,4)$ & \\
\hline
\end{tabular}

(continua) 
Tabla 2 (continuación)

\begin{tabular}{|c|c|c|c|c|c|c|}
\hline \multirow[t]{3}{*}{ Características } & \multicolumn{2}{|c|}{ Antioquia (\%) [n = 588] } & \multirow{3}{*}{$\begin{array}{l}\text { Valor de p } \\
\text { unilateral }\end{array}$} & \multicolumn{2}{|c|}{ Bogotá (\%) [n = 423] } & \multirow{3}{*}{$\begin{array}{l}\text { Valor de } p \\
\text { unilateral }\end{array}$} \\
\hline & Casos & Controles & & Casos & Controles & \\
\hline & {$[n=196]$} & {$[n=392]$} & & {$[n=141]$} & {$[n=282]$} & \\
\hline Índice de masa corporal & & & 0,21 & & & 0,25 \\
\hline$>28$ & $57(36,1)$ & $131(41,1)$ & & $19(13,5)$ & $25(8,9)$ & \\
\hline $24,0-27,9$ & $44(27,8)$ & $97(30,4)$ & & $37(26,2)$ & $73(25,9)$ & \\
\hline$<24$ & $57(36,1)$ & $91(28,5)$ & & $85(60,3)$ & $184(65,2)$ & \\
\hline Diagnóstico inoportuno & $16(8,2)$ & $9(2,3)$ & 0,00 & $34(24,1)$ & $44(15,6)$ & 0,01 \\
\hline Diagnóstico incorrecto & $23(11,8)$ & $34(8,7)$ & 0,11 & $46(32,6)$ & $72(25,5)$ & 0,06 \\
\hline Tratamiento inoportuno & $45(23,1)$ & $54(13,8)$ & 0,00 & $49(28,4)$ & $95(20,2)$ & 0,41 \\
\hline Tratamiento incorrecto & $59(30,3)$ & $82(20,9)$ & 0,00 & $40(28,4)$ & $57(20,2)$ & 0,03 \\
\hline Calidad de la atención & & & 0,02 & & & 0,39 \\
\hline Sin fallas & $128(65,6)$ & $290(74,0)$ & & $68(48,2)$ & $140(49,6)$ & \\
\hline Al menos una falla & $67(34,4)$ & $102(26,0)$ & & $73(51,8)$ & $142(50,4)$ & \\
\hline Dificultades transporte & $0(0,0)$ & $1(0,3)$ & 0,13 & $8(5,7)$ & $8(2,8)$ & 0,07 \\
\hline Dificultades administrativas & $6(3,1)$ & $3(0,8)$ & 0,40 & $10(7,1)$ & $24(8,5)$ & 0,30 \\
\hline Falta de suministros & $5(2,6)$ & $3(0,8)$ & 0,09 & $5(3,5)$ & $9(3,2)$ & 0,42 \\
\hline
\end{tabular}

DE: deviación estándar.

* Educación de la gestante: no se tiene esta información para el 47\% de los casos y el 55\% de los controles en Antioquia;

** No se obtuvieron datos para aproximadamente el $40 \%$ de casos y controles.

oportuno y dedicado a todas y cada una de las pacientes.

En la ocurrencia de la mortalidad materna existen igualmente problemas relacionados con la calidad de la atención 21,22,23. El cuidado obstétrico de calidad es un factor clave para reducir la mortalidad materna y la morbilidad obstétrica externa, y la evaluación del proceso de atención y la toma de decisiones con base en los hallazgos son pilares en la prevención de un nuevo caso de mortalidad materna 24 . En nuestro estudio, el diagnóstico inoportuno se asoció con un incremento en el riesgo de sufrir una morbilidad obstétrica externa en Antioquia y con una tendencia importante, aunque no alcanzó significación estadística, en Bogotá. El diagnóstico temprano permite tomar medidas de tratamiento en un momento de la evolución de la complicación, en la cual aún se puede recuperar la condición clínica de la mujer. Si se permite el deterioro de la condición clínica, es más probable que los tratamientos ya no tengan posibilidad de actuar, porque se puede estar ante condiciones clínicas irreversibles. Un tratamiento correcto en un estado crítico avanzado por un diagnóstico no oportuno tiene mucha menos probabilidad de ser efectivo, lo cual es la probable explicación a la falta de efecto del tratamiento incorrecto hallado en esta investigación.

El diagnóstico inoportuno sugiere la presencia de limitaciones para identificar a tiempo la presencia de una morbilidad obstétrica externa o una subvaloración de los cuadros clínicos. A su vez, estas limitaciones se pueden deber a falta de competencias del personal de la salud, al tiempo reducido para atender un número importante de mujeres gestantes, a la multiplicidad de funciones que tiene que cumplir el personal en los servicios obstétricos como hacer consulta, cuidar trabajos de parto, atender partos y hacer cirugías. Igualmente, la subvaloración de la gravedad del caso podría ser explicada por un problema de actitud del personal de la salud.

El hecho de que los factores asociados, y las magnitudes de los efectos encontrados sean diferentes entre las dos regiones estudiadas, respalda la necesidad de hacer estudios locales y específicos, porque las intervenciones podrían ser diferentes dependiendo de las condicionantes identificadas.

Una dificultad para la comparación de nuestros resultados con otros estudios es que no existe uniformidad en la definición de la morbilidad obstétrica externa en la literatura universal, y para el momento de la implementación de esta investigación aún no se contaba con el consenso de la Organización Mundial de la Salud (OMS). La definición utilizada en esta investigación está centrada en criterios clínicos de una enfermedad específica y de disfunción de órganos o sistemas, lo cual permite una mayor facilidad de interpretación y de recolección de casos. Está decisión 
Asociación entre la presencia de morbilidad obstétrica grave y características demográficas y de la atención.

\begin{tabular}{|c|c|c|}
\hline Características & Antioquia & $\begin{array}{c}\text { Bogotá } \\
\text { OR (IC95\%) }\end{array}$ \\
\hline Pago per cápita (vs pago por evento) & $2,05(0,00-1,11)$ & $1,31(0,80-2,13)$ \\
\hline \multicolumn{3}{|l|}{ Régimen } \\
\hline Contributivo + especial & Referencia & Referencia \\
\hline Subsidiado & $0,39(0,12-1,30)$ & $0,29(0,16-0,54)$ \\
\hline No aseguradas & $0,35(0,50-2,44)$ & $0,29(0,15-0,57)$ \\
\hline Zona rural (vs urbana) & $1,51(0,24-9,50)$ & \\
\hline \multicolumn{3}{|l|}{ Edad (años) } \\
\hline 30 & Referencia & Referencia \\
\hline $20-29$ & $1,25(0,27-5,72)$ & $1,14(0,57-2,28)$ \\
\hline Menores de 19 & $0,57(0,14-2,37)$ & $1,34(0,72-2,51)$ \\
\hline \multicolumn{3}{|l|}{ Numero de controles prenatales } \\
\hline 5 o más controles & Referencia & Referencia \\
\hline 1-4 controles & $2,14(0,71-6,48)$ & $1,26(0,73-2,17)$ \\
\hline Sin control prenatal & $1,60(0,97-26,57)$ & $2,15(1,04-4,45)$ \\
\hline \multicolumn{3}{|l|}{ Inicio del control prenatal (semanas) } \\
\hline Antes de las 13 & Referencia & Referencia \\
\hline Entre $13-20$ & $0,55(0,16-1,88)$ & $1,83(1,03-3,27)$ \\
\hline Despúes de las 20 & $0,63(0,13-3,01)$ & $1,34(0,57-3,19)$ \\
\hline Diagnóstico oportuno & $3,67(0,43-31,63)$ & $1,42(0,72-2,81)$ \\
\hline Diagnóstico correcto & $1,28(0,20-8,01)$ & $1,35(0,77-2,38)$ \\
\hline Tratamiento oportuno & $1,36(0,36-5,19)$ & $0,76(0,44-1,32)$ \\
\hline Tratamiento correcto & $0,49(0,15-1,61)$ & $1,45(0,76-2,77)$ \\
\hline \multicolumn{3}{|l|}{ Educación de la gestante } \\
\hline Técnica o universitaria & Referencia & Referencia \\
\hline Primaria & $0,80(0,22-2,97)$ & $2,55(1,13-5,75)$ \\
\hline Secundaria & $0,56(0,18-1,78)$ & $0,92(0,54-1,56)$ \\
\hline
\end{tabular}

IC95\%: entervalo de confianza al 95\%; OR: odds ratio.

* Modelo ajustado por las variables reportadas en metodología. Se excluyó la diabetes, debido al escaso número de casos. Nota: en el modelo de Bogotá se excluyó la zona (urbana/rural) debido a que menos del 2\% reportó zona rural.

está acorde con la recomendación de Ronsman \& Filippi 24 de utilizar una definición pertinente para cada región. Una limitación de esta investigación es que no se realizó una entrevista directa a las pacientes para conocer su percepción de la calidad de la atención recibida, ni al personal de la salud para conocer la percepción que ellos tienen de los factores que permitieron que se presentara la morbilidad obstétrica externa.
En conclusión, los factores asociados a la morbimortalidad materna son diferentes, de acuerdo con la población estudiada, lo que sugiere la necesidad de hacer estudios locales para poder identificar los factores determinantes propios y tomar las decisiones pertinentes para mejorar la situación particular. 


\section{Colaboradores}

F. J. Yepes dirigió el estudio a nivel nacional, participó en el diseño, en el análisis de los datos, la escritura y revisión del documento, y asume plena responsabilidad por la información que se publica. J. G. Gómez dirigió el estudio en Antioquia, participó en el diseño, en el análisis de los datos, la escritura y revisión del documento, y asume plena responsabilidad por la información que se publica. J. J. Zuleta participó en el diseño, en la coordinación del trabajo de campo en Antioquia, en el análisis de los datos, la escritura y revisión del documento, y asume plena responsabilidad por la información que se publica. J. L. Londoño participó en el diseño del estudio, realizó el diseño de la muestra, dirigió el análisis de los datos, participó en la escritura y revisión del documento, y asume plena responsabilidad por la información que se publica. J. L. Acosta-Reyes asumió el trabajo de campo en Antioquia, tuvo a su cargo el procesamiento de los datos y su análisis preliminar, participó en la escritura y revisión del documento, y asume plena responsabilidad por la información que se publica. L. H. Sánchez-Gómez participó en el diseño del estudio, dirigió la capacitación del equipo de campo, participó en el trabajo de campo en Bogotá, en el análisis de los datos, la escritura y revisión del documento, y asume plena responsabilidad por la información que se publica. M. L. Ramírez coordinó el trabajo de campo en Bogotá, hizo el control de calidad de los datos, diseñó la bases de datos, participó en la escritura y revisión del documento, y asume plena responsabilidad por la información que se publica.

\section{Agradecimientos}

A la enfermera María Patricia González de la Secretaría Distrital de Salud quien facilitó la vinculación de la Secretaría y de todo el equipo de apoyo local. A las enfermeras María Teresa Espitia, Sonia Marcha, Diana Gracia, Catherine Rodríguez, Xiomara Fernández y Adriana Guaca, responsables del trabajo de campo en Bogotá, así como a las directivas y profesionales de los hospitales de Medellín y Bogotá. IDRC, ASSALUD, Centro Nacer de Salud Sexual y Reproductiva y Secretaría de Salud de Bogotá.

\section{Referencias}

1. Hill K, Thomas K, AbouZahr C, Walker N, Say L, Inoue $\mathrm{M}$, et al. Estimates of maternal mortality worldwide between 1990 and 2005: an assessment of available data. Lancet 2007; 370:1311-9.

2. Say L, Souza JP, Pattinson RC; WHO Working Group on Maternal Mortality and Morbidity Classifications. Maternal near miss: towards a standard tool for monitoring quality of maternal health care. Best Pract Res Clin Obstet Gynaecol 2009; 23:287-96.

3. Mantel GD, Buchmann E, Rees H. Pattinson RC. Severe acute maternal morbidity: a pilot study of a definition for a near-miss. Br J Obstet Gynaecol 1998; 105:985-90.

4. Zwart JJ, Richters JM, Öry F, De Vries JIP, Bloemenkamp KWM, Van Roosmalen J. Severe maternal morbidity during pregnancy, delivery and puerperium in the Netherlands: a nationwide populationbased study of 371000 pregnancies. BJOG 2008; 115:842-50.

5. Paruk F, Moodley J. Severe obstetric morbidity. Curr Opin Obstet Gynecol 2001; 13:563-8.

6. Pattinson RC, Hall M. Near misses: a useful adjunct to maternal death enquiries. Br Med Bull 2003; 67:231-43.

7. Vandecruys HI, Pattinson RC, Macdonald AP, Mantel GD. Severe acute maternal morbidity and mortality in the Pretoria Academic Complex: changing patterns over 4 years. Eur J Obstet Gynecol Reprod Biol 2002; 102:6-10.

8. Ronsmans C. Severe acute maternal morbidity in low-income countries. Best Pract Res Clin Obstet Gynaecol 2009; 23:305-16.

9. Profamilia. Encuesta Nacional de Demografía y Salud 2010. http://www.profamilia.org.co/en cuestas/Profamilia/Profamilia/images/stories/ PDF-capitulos/Capitulo-14.pdf (accedido el 24/ Mar/2014).

10. World Health Organization. Trends in maternal mortality: 1990 to 2008. Estimates developed by WHO, UNICEF, UNFPA and the World Bank; 2010. http://whqlibdoc.who.int/publications/ 2010/9789241500265_eng.pdf (accedido el 24/ Mar/2014).

11. Ministerio de la Protección Social. Decreto 4.747 de 2007, diciembre 7. http://www.minsalud.gov. co/Normatividad/DECRETO\%204747\%20DE\%20 2007.pdf (accedido el 24/Mar/2014).

12. Gosden T, Forland F, Kristiansen I, Sutton M, Leese B, Giuffrida A, et al. Capitation, salary, fee-for-service and mixed systems of payment: effects on the behaviour of primary care physicians. Cochrane Database Syst Rev 2000; (3):CD002215.

13. Londoño JL. Metodología de la investigación epidemiológica. 3ạ Ed. Bogotá: Manual Moderno; 2004.

14. Shen FR, Liu M, Zhang X, Yang W, Chen YG. Factors associated with maternal near-miss morbidity and mortality in Kowloon Hospital, Suzhou, China. Int J Gynaecol Obstet 2013; 123:64-7.

15. Geller SE, Rosenberg D, Cox SM, Brown ML, Simonson L, Driscoll CA, et al. The continuum of maternal morbidity and mortality: factors associates with severity. Am J Obstet Gynecol 2004; 191:939-44. 
16. García FM. Los determinantes de la calidad de la atención en salud: el caso del control prenatal. Coyunt Económica 2009; 39:61-97.

17. Ministerio de la Protección Social. Ley 1.438 de 2011: por medio del cual se reforma el Sistema General de Seguridad Social en Salud y se dictan otras disposiciones. Diario Oficial 2011; jan.

18. Congreso de Colombia. Ley Estatutaria no 1.751 de 2015 por la cual se regula el derecho fundamental a la salud y se dictan otras disposiciones. Diário Oficial 2015; feb.

19. Bouvier-Colle M, Joud OE, Varnoux N, Goffinet F, Alexander S, Bayoumeu F, et al. Evaluation of the quality of care for severe obstetrical haemorrhage in three French regions. BJOG 2001; 108:898-903.

20. Lewis G. Saving mothers' lives: reviewing maternal deaths to make motherhood safer - 2003-2005. The Seventh Report on Confidential Enquiries into Maternal Deaths in the United Kingdom. London: Confidential Enquiry into Maternal and Child Health; 2007.
21. Cantwell R, Clutton-Brock T, Cooper G, Dawson A, Drife J, Garrod D, et al. Saving mothers' lives: reviewing maternal deaths to make motherhood safer: 2006-2008. The Eighth Report of the Confidential Enquiries into Maternal Deaths in the United Kingdom. BJOG 2011;118 Suppl 1:1-203.

22. Instituto Nacional de Salud. Protocolo de vigilancia en salud pública: morbilidad materna extrema. Bogotá DC: Instituto Nacional de Salud; 2014.

23. Vélez-Álvarez G, Gómez-Dávila J, Zuleta-Tobón J. Análisis de las muertes maternas por hemorragia en el departamento de Antioquia, Colombia. Años 2004 y 2005. Rev Colomb Obstet Ginecol 2006; $57: 147-55$

24. Ronsmans C, Filippi V. Reviewing severe maternal morbidity: learning from survivors of life-threatening complications. In: World Health Organization, editor. Beyond the numbers reviewing maternal deaths and complications to make pregnancy safer. Geneva: World Health Organization; 2004. p. 103-23.

\section{Resumo}

O presente estudo objetiva identificar se as formas de pagamento e regimes de seguros de saúde estão associados com a mortalidade materna e morbidade materna grave. Trata-se de um estudo caso-controle de base hospitalar em duas regiões da Colômbia entre 2009-2011. Os dados foram obtidos através da história clínica de cada gestante. Foi utilizada a regressão logística não condicionada. A mostra incluiu 1.011 pacientes: 337 casos e 674 controles. Nenhum componente de qualidade foi estatisticamente significativo em ambas as regiões. Em Bogotá, o risco de complicações obstétricas foi significativamente maior no Regime Contributivo do que no Subsidiado e em mulheres sem seguro de saúde. Em Antioquia, embora existam associações similares, não foram estatisticamente significativas. Tanto em Antioquia quanto em Bogotá não se encontraram diferenças significativas de morbilidade por regime de pagamento. Foram encontrados diferentes fatores associados à morbimortalidade materna, segundo a população estudada, o que sugere a necessidade de estudos locais para identificar fatores determinantes próprios e tomar decisões adequadas neste contexto.

Seguro Saúde; Complicações na Gravidez; Mortalidade Materna
Recibido el 26/Mar/2015

Versión final presentada el 04/Feb/2016

Aprobado el 11/Mar/2016 